\title{
A Systematic Review on the Anxiolytic Effects of Aromatherapy in People with Anxiety Symptoms
}

\author{
Yuk-Lan Lee, BSc,' Ying Wu, BSc, Hector W.H. Tsang, PhD, ${ }^{1}$ Ada Y. Leung, MA, and W.M. Cheung, PhD²
}

\begin{abstract}
Purpose: We reviewed studies from 1990 to 2010 on using aromatherapy for people with anxiety or anxiety symptoms and examined their clinical effects.

Methods: The review was conducted on available electronic databases to extract journal articles that evaluated the anxiolytic effects of aromatherapy for people with anxiety symptoms.

Results: The results were based on 16 randomized controlled trials examining the anxiolytic effects of aromatherapy among people with anxiety symptoms. Most of the studies indicated positive effects to quell anxiety. No adverse events were reported.

Conclusions: It is recommended that aromatherapy could be applied as a complementary therapy for people with anxiety symptoms. Further studies with better quality on methodology should be conducted to identify its clinical effects and the underlying biologic mechanisms.
\end{abstract}

\section{Introduction}

A NXIETY IS A PSYCHOLOGIC and physiologic state characterized by cognitive, somatic, emotional, and behavioral components. ${ }^{1}$ About $4 \%-6 \%$ of the global population suffer from various forms of anxiety disorders with such symptoms as high blood pressure, elevated heart rate, sweating, fatigue, unpleasant feeling, tension, irritability, and restlessness. ${ }^{2}$ If untreated, $40 \%-50 \%$ of the patients would progress to depression and have suicidal thoughts. ${ }^{3}$ The symptoms bring huge negative impact to their families, social, and occupational roles. National statistics show that in the United States, anxiety disorders incurred $\$ 46.6$ billion direct and indirect costs each year, which constituted nearly one third of the nation's total mental health expenses. ${ }^{4}$

Pharmacologic and psychologic treatments have remained the conventional interventions to treat anxiety disorders for the past 30 years. ${ }^{5}$ However, pharmacologic treatment causes many side-effects. For example, benzodiazepine, a popular medication with powerful anxiolytic effects, has been well known for its side-effects including sedation, muscle relaxation, headache, and ataxia. ${ }^{6}$ These side-effects significantly reduce adherence of the patients. Another problem is that some anti-anxiety drugs are potentially addictive. Reoccurrence of anxiety symptoms will result from removal of the drugs. ${ }^{7}$ Psychologic treatment, especially cognitive behavior therapy, is the main alternative to drug therapy. ${ }^{5}$
Unfortunately, the effect is not at all conclusive based on available information. ${ }^{8}$

Recently, a remarkable increase in the use of complementary and alternative medicine (CAM) around the globe is evidenced. Aromatherapy is a commonly used CAM that has long been regarded as a popular means of treatment for anxiety. It involves the therapeutic use of essential, aromatic oils, commonly combined with therapeutic massage and excitation of the olfactory system, to induce relaxation and thus quell certain anxiety symptoms. ${ }^{9}$ Aromatherapy is claimed to be beneficial to the mental, psychologic, spiritual, and social aspects, although they are less quantitatively measurable. With respect to safety, it is reported that that aromatherapy is relatively free of adverse effects compared with conventional drugs. ${ }^{10}$

Unlike conventional medicine, the effectiveness of aromatherapy remains unclear and is still under intensive research. To date, there is only one relevant review on aromatherapy for depression. ${ }^{11}$ Although depression and anxiety are usually co-occurring, a separate systematic review on the anxiolytic effects of aromatherapy is still needed. To date, there has not been a systematic review on the anxiolytic effects of aromatherapy. The purpose of the current review is to fill the gap by unraveling the effectiveness of aromatherapy on relieving anxiety symptoms. Based on extant literature, the evidence was integrated so as to aid in gaining a better understanding on the clinical use of

\footnotetext{
${ }^{1}$ Neuropsychiatric Rehabilitation Laboratory, Department of Rehabilitation Sciences, The Hong Kong Polytechnic University, Hong Kong.

${ }^{2}$ Faculty of Education, The University of Hong Kong, Hong Kong.
} 
aromatherapy as a CAM to treat people suffering from anxiety symptoms.

\section{Methods}

\section{Literature search}

Studies used in this review were extracted from MEDLINE $^{\circledR}$, Social Sciences Citation Index, Science Citation Index, Psyinfo, PsyARTICLES, Journals@Ovid, MD Consult, ScienceDirect, EBSCOHOST, and Handbook of Psychiatry, from 1990 to 2008, using keywords "anxiety disorder," "anxiety," "anxious symptom" or "anxiolytic effects" and "aromatherapy", "aroma," or "essence oil." Only English publications were included. Potential titles were retrieved for the second stage of review. The titles and the available abstracts were then independently reviewed. Neither of the reviewers was blind to the author name, institution, and/or the journal.

The target was to extract randomized controlled trials (RCT) that used aromatherapy as the intervention to relieve anxiety symptoms that were measured by validated inventories. A study was operationally defined as a RCT in this review if the allocation of participants to treatment and comparison groups was reported to be randomized, the sample size was not less than 10 in each arm, the participants were aged 18 or older, and anxiety was included as the outcome measure. Studies that did not use any type of comparison group, were qualitative in nature, and were systematic review or meta-analysis were excluded.

\section{Quality assessment}

Studies selected based on the above criteria, and methods were evaluated for methodological vigor. Guidelines set out by Glasziou et al. ${ }^{12}$ were followed, and the quality of the studies was assessed by reviewing whether they fulfilled the criteria of control randomization, allocation concealment, intention to treat, and blindedness. Adequately concealed RCT means that the trial had a clear description of its allocation procedure, central randomization, and allocation from site apart from the study area and/or blinding allocation procedure. An RCT is considered to have used intention-totreat analysis if all the randomized participants were analyzed with no differences between the treatment allocation before and after application of treatment procedure. ${ }^{13} \mathrm{~A}$ study was classified as "single blind" if the outcome measure was conducted by an assessor who was blind to the treatment allocation while the participants were not blind to the treatment. A study was classified as "double blind" if both the assessor of outcome measure and the participants were blind to the treatment allocation. A study was considered not blind if neither the assessor nor the participants were blind to the outcome measure and treatment allocation, respectively. ${ }^{14}$

\section{Data synthesis}

Due to heterogeneity of the study populations, psychometric instruments, and intervention trials, quantitative analysis on the effect size was not performed. However, qualitative analysis using the Sjösten method ${ }^{15}$ was employed to classify interventions as having positive, negative, or no effect as determined by whether significant differences in anxiety symptoms were observed in at least one of the outcome measures between the study groups.

\section{Results}

\section{Study description}

The numbers of citations returned from the database search were 70, 73, and 42 for MEDLINE, SSCI+ SCI, and others (Psyinfo, PsyARTICLES, Journals@Ovid, MD Consult, ScienceDirect, EBSCOHOST, and Handbook of Psychiatry), respectively, in March 2010. Fifty-two (52) relevant publications were extracted for further evaluation. After abstract screening at the first stage and full-text screening at the second stage, 16 studies met the inclusion criteria. Figure 1 summarizes the selection process of the eligible RCTs.

Table 1 summarizes the methods and results of the 16 qualified RCTs. The total number of subjects involved was 25,377 , in which the female-to-male ratio was $24,887: 490$. The age of the participants ranged from 18 to 90 years $(M=47.77)$. All subjects suffered from obvious anxiety symptoms. Patients receiving palliative care were reported in three studies. ${ }^{16-18}$ Healthy volunteers with experimentally induced stress were the second most popular client types that were reported in two studies. ${ }^{19,20}$ Other studies recruited different types of clients, including mothers in labor, postpartum mothers, women prepared for surgical abortion, participants prepared for endoscopy procedure, patients prepared for dental procedures, patients with cancer during radiotherapy, nursing students attended for stressful surgical disease examination, patients with cancer with clinically diagnosed with anxiety/depression, patients with moderate and severe dementia, patients in hematology transplant unit, and patients primarily diagnosed with generalized anxiety disorder. The types of aromatherapy administration in the RCTs included aromatherapy massage, inhalation, tablet intake, and footbath. The intervention duration of aromatherapy massage ranged from 20 minutes to 1 hour, and the duration of inhalation ranged from 5 minutes to 1 hour. The most commonly used essential oil used in these studies was lavender. ${ }^{17,19,21-25}$

\section{Outcomes}

Only 14 studies adopted a control group with a compatible "conventional therapy" or a "placebo," and the remaining two studies used a control group with "no active treatment." Fourteen (14) studies reported positive findings as to the anxiolytic effects of aromatherapy; ${ }^{16-22,24,26-31}$ while the remaining two studies ${ }^{23,25}$ reported no effect of the aromatherapy toward anxiety symptoms. In comparing changes and improvement between the aromatherapy and control groups providing no active interventions, the subjects who received aromatherapy usually showed better outcomes than those in the control groups. However, when comparing the effect of aromatherapy to a conventional treatment or a placebo (e.g., massage with carrier oil, inactive coated tablets, benzodiazepine, sniff a hair conditioner, music therapy, etc.), the results were inconsistent. Seven (7) studies indicated that aromatherapy had benefits that were superior to conventional therapy or placebo. ${ }^{19-22,24,26,27}$ In contrast, five studies ${ }^{17,18,28-30}$ reported that the therapeutic effects between massage group and aromatherapy group were similar. One 


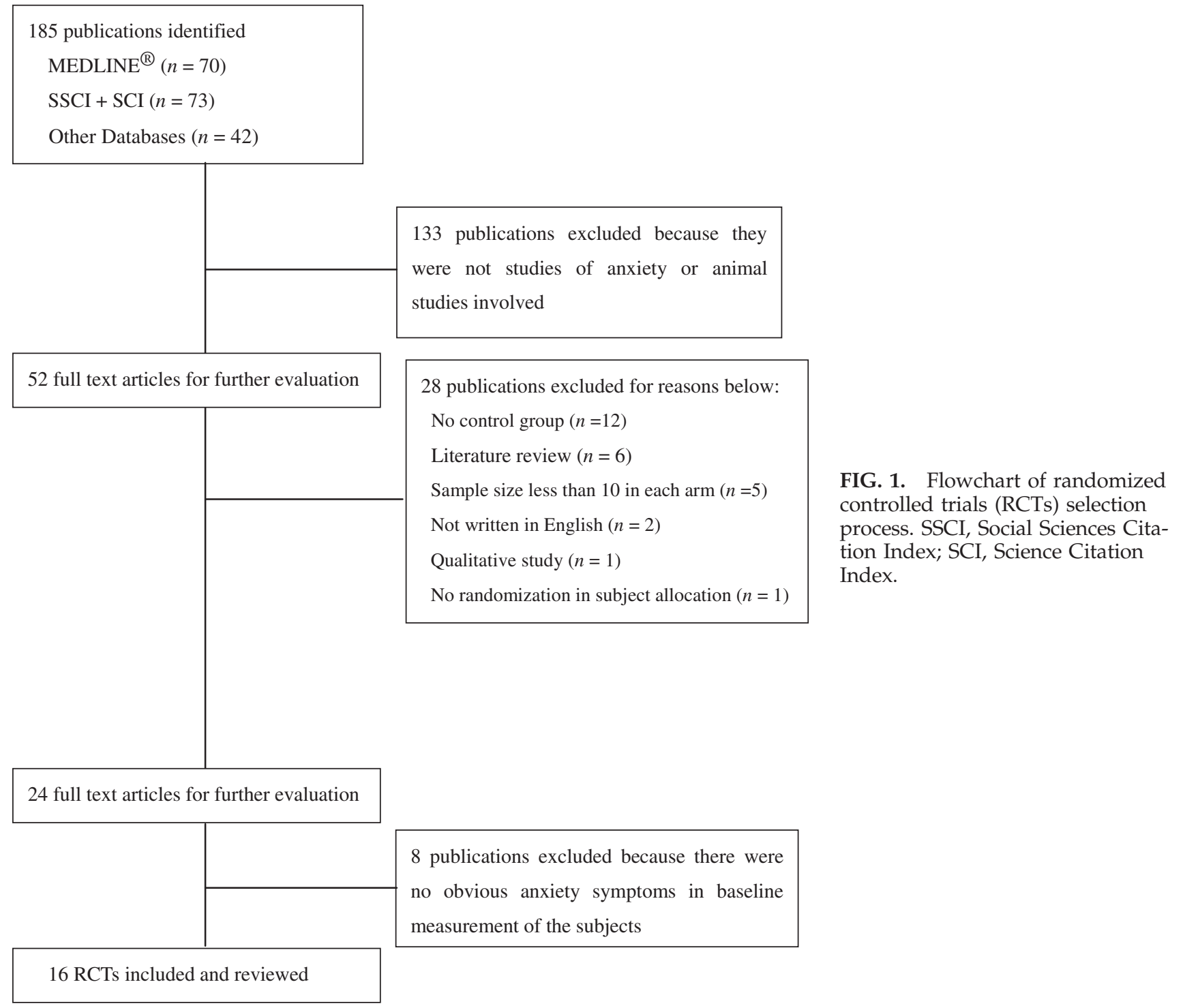

(1) study ${ }^{16}$ reported that the anxiolytic effect of massage with carrier oil only was significantly better than those receiving massage with essential oil. One study reported that an oral lavender oil capsule is as effective as lorazepam, a benzodiazepine, in adults with generalized anxiety disorder. ${ }^{31}$ Two (2) studies ${ }^{26,28}$ had follow-up data after the treatment. Both of them suggested that no long-term effect was evidenced, and aromatherapy did not appear to confer benefit on anxiety.

\section{Study quality}

All studies applied random allocation. Seven of the 16 studies nevertheless had no clear description on the randomization procedures. ${ }^{16,17,19,22,23,25,29}$ Only one study ${ }^{21}$ described the concealment of allocation procedure, but the description was inadequate. Double-blindedness during outcome assessment was described in three studies ${ }^{20,30,31}$ and single-blindedness in six studies. ${ }^{21,22,26,28}$ The massage therapists in the studies did not belong to the research team and did not need to conduct assessments of the subjects in order to ensure the double-blindedness. Seven (7) of the 16 studies did not mention whether blinding techniques were applied. ${ }^{16-19,23,27,29}$ Intention-to-treat analysis was employed in 11 studies. ${ }^{19-24,26-30}$ One (1) study ${ }^{16}$ mentioned the high dropout rate due to the long research period. In addition, the number of subjects recruited for individual studies varied greatly, from 24 to 23,857 .

\section{Pooled effect size}

State Anxiety Inventory (SAI) was commonly used in the 16 reviewed studies. Pooled effect size of the outcome measure of SAI is conducted from pre- and post- means and standard deviations of the control and treatment groups of three studies. ${ }^{18,26,27}$ Other studies are not included because corresponding authors could not be contacted for further information. Pooled effect size is shown in Table 2. 


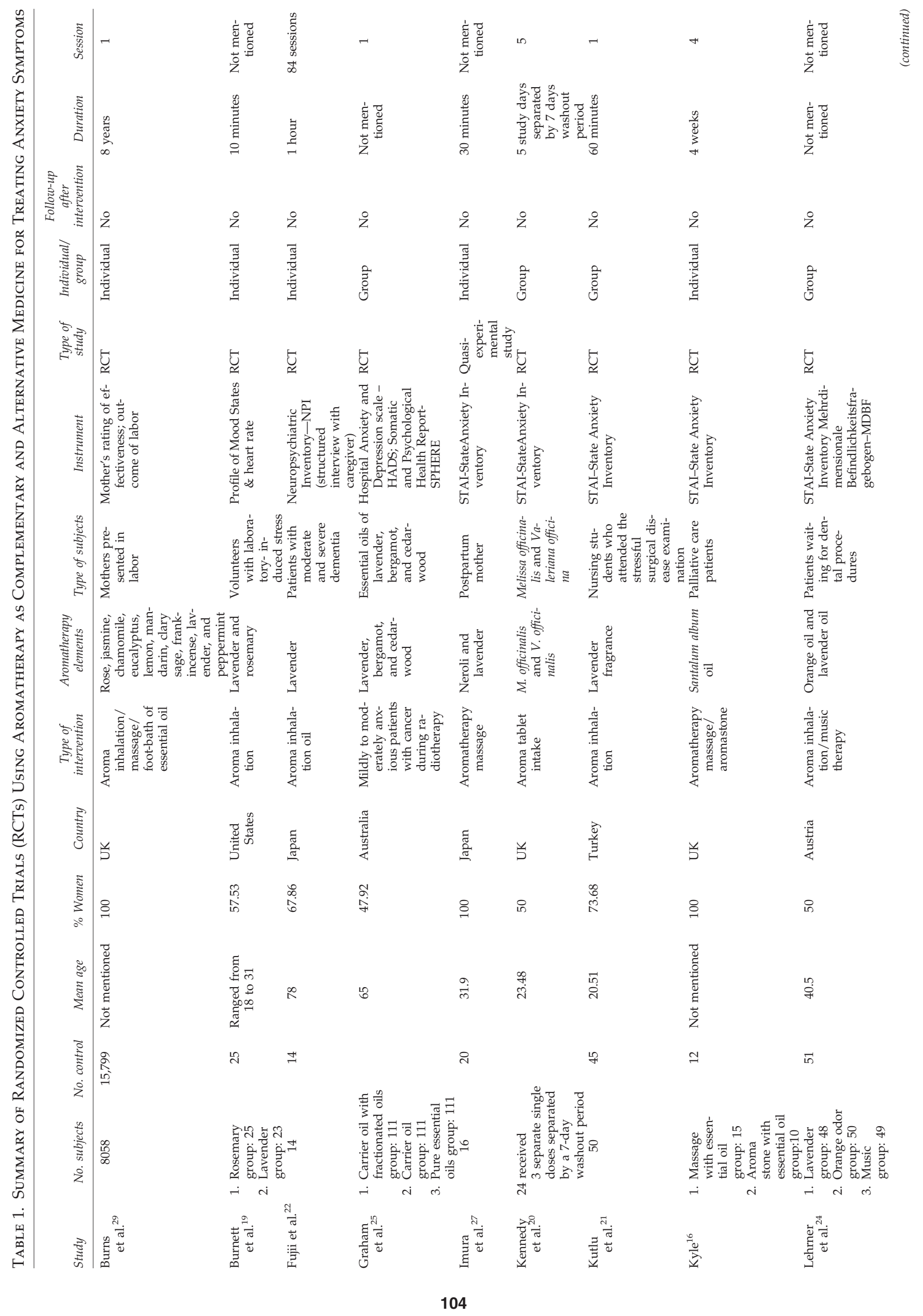




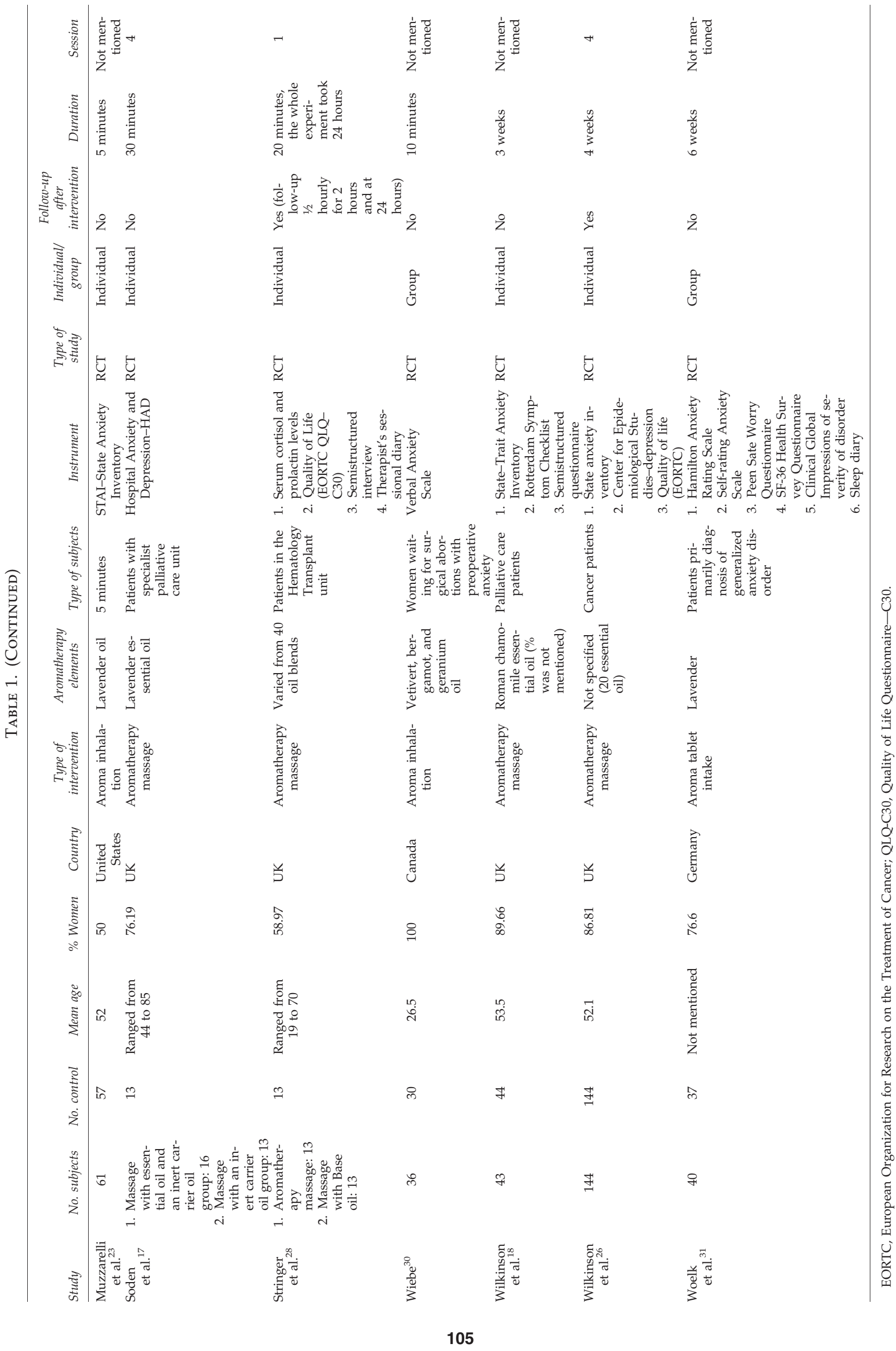


Table 2. Pooled Effect Size of Aromatherapy Massage Studies with State AnXiety InVEntory Outcome Measure

\begin{tabular}{llc}
\hline Study & Effect size & Pooled effect size \\
\hline Imura et al. ${ }^{27}$ & -1.617 & \\
Wilkinson et al. $^{18}$ & -0.0708 & \\
Wilkinson et al. $^{26}$ & -0.5030 & -0.5103 \\
\hline
\end{tabular}

\section{Discussion}

Aromatherapy is the most commonly used CAM for treating anxiety symptoms around the world. ${ }^{32}$ Our review reveals that aromatherapy shows a positive anxiolytic effect for patients with anxiety symptoms and more importantly, it is a safe intervention, and no participants in the studies reported any adverse effects. However, drawing conclusions on the effectiveness of aromatherapy for relieving anxiety symptoms should be done with care and caution.

This review shows that there are insufficient clinical trials examining the effects of aromatherapy among people with anxiety disorders as the primary illness. All of the 16 studies in our review in fact examined the effects of aromatherapy on secondary anxiety symptoms in various types of participants, including people with cancer, dementia, postpartum mothers, and healthy volunteers. In addition, the anxiety levels of the participants differed significantly from mild to moderate in the pretests. The effectiveness of aromatherapy could hardly be compared among participants with different levels of anxiety. Improvement in anxiety symptoms among participants with mild anxiety tended to be insignificant. In contrast, participants with high levels of psychologic distress responded better to aromatherapy interventions. ${ }^{17}$ To improve the quality of research efforts in the future, the level of severity of anxiety can be raised to moderate or greater in the recruitment of participants to assure the validity of the results.

The Spielberger State-Trait Anxiety Inventory, adopted as the assessment tool on evaluating anxiety levels in eight studies, was the most commonly used among the 16 studies. It is reported to be a reliable and valid self-rating assessment in research and clinical practice. ${ }^{33}$ The meta-analysis of pooled effect size in the current study shows that aromatherapy massage has a median treatment effect for anxiety. However, it should be noted that the pool effect size is obtained from three studies with different essential oils and treatment duration.

As to the administration of aromatherapy, six studies employed aromatherapy massage and seven studies used the method of inhalation. Other modalities such as internal or oral application and footbath were mentioned in three studies. Yim et al. ${ }^{11}$ and Imura et al. ${ }^{27}$ raised the question of whether the effect was due to the aromatherapy alone or its interaction effect with massage. In this review, different implementations of aromatherapy have made the effect noncomparable and undifferentiated. It is obvious that inhalation involved purely olfactory stimulation, internal intake involved both olfactory stimulation and body metabolism, and footbath and aromatherapy massage consisted of olfactory stimulation, somatosensory stimulation, and tactile stimulation. Four (4) studies made comparisons between massage and aromatherapy massage. Three (3) of them stated a tendency for aromatherapy massage to be slightly more effective than the "placebo." One (1) reported that massage alone had slightly better anxiolytic effect than aromatherapy massage. However, the differences were modest and could have been attributed to flaws in the study design. It is therefore important to determine the best modalities of aromatherapy in future studies. Comparison between inhalation, aromatherapy massage, oral intake, and a control group with a compatible "conventional treatment/ placebo" in future studies will be necessary to rule out the effects of nonspecific factors and to unify the modalities of aromatherapy.

The quality of the studies' design prevented drawing firm valid conclusions as to the clinical efficacy of aromatherapy. The size of samples varied largely in the present studies. Except for one study with a large number of participants $(n=23,857)$, five studies used only a small sample size $(n=24, n=28, n=34, n=36, n=39)$. Also, the gender distribution among the participants was uneven, with the female subjects outnumbering $(n=24,887)$ the male subjects $(n=490)$ on the whole $(n=25,377)$ among the five reviewed studies. The reason is that one of the reviewed studies with the largest sample size $(n=23,857)$ involved only female subjects who were in fact mothers in labor. Other than this study, the distribution of gender of other studies was even. Further research should employ comparable numbers of male and female participants. Studies also showed significant differences in the duration of treatment. One (1) study lasted only 5 minutes, while two studies lasted 60 minutes. It is uncertain whether the duration of aromatherapy treatment between studies would have affected the outcomes. Furthermore, the studies adopted different types of essential oil. It is unknown whether the effects were due to a specific essential oil (e.g., lavender, etc.) or the general properties of various essential oils. Although our studies were all RCTs in nature, there were obvious methodological limitations. To provide further evidence for advocating aromatherapy as an effective complementary or alternative treatment to reduce anxiety symptoms, studies with stricter and more vigorous procedures in allocation concealment and blinding should be implemented. Compliance to the therapy should be examined more thoroughly by intention-to-treat analysis.

Notwithstanding the promising therapeutic effects of aromatherapy, there has not been literature that could provide a sound biologic rationale for the use of aromatherapy as a complementary and alternative intervention. The psychobiologic mechanism underlying the anxiolytic effect remains unclear. According to previous research, ${ }^{34}$ $\gamma$-aminobutyric acid (GABA), one of the brain neurotransmitters, has an inhibitory effect upon the nervous system and hence may be used to calm the overstimulated nervous system under tension and stress. Previous research efforts $^{35,36}$ have suggested that some essential oils (e.g., lavender, etc.) worked similarly to diazepam, which acts as the agonist of GABA. One of the current authors' reviewed studies $^{31}$ also stated that an oral lavender oil capsule, silexan, is as effective as lorazepam, which is a commonly used benzodiazepine. Some studies ${ }^{37}$ hypothesized that the anxiolytic effects may be due to the retrieval of pleasant 
memories by particular smells associated with some essential oils. The unclear biologic mechanisms explaining how aromatherapy reduces anxiety symptoms leave room for further research.

\section{Conclusions}

As generally all of the 16 reviewed studies showed a positive result of aromatherapy on anxiety, it is recommended that aromatherapy could be applied as a complementary therapy for people with anxiety symptoms. Although there is no conclusive evidence to show lasting effects of aromatherapy for treating anxiety, it may best be considered as a safe and pleasant intervention for those who can afford it and are prepared to pay for it.

\section{Disclosure Statement}

No competing financial interests exist.

\section{References}

1. Seligman MEP, Walker EF, Rosenhan DL. Abnormal Psychology. New York: W.W. Norton \& Company, 2001.

2. Smith M. Anxiety Attacks and Disorders: Guide to the Signs, Symptoms, and Treatment Options. Help Guide website. June 2008. Online document at: www.helpguide.org/ mental/anxiety_types_symptoms_treatment.htm Accessed March 3, 2009.

3. Treating depression and anxiety in primary care. Prim Care Companion J Clin Psychiatry 2008;10:145-152.

4. National Mental Health Association. Online document at: www.capefearhealthyminds.org/ April 27, 2009.

5. Schmidt NB, Keough ME, Hunter LR, Funk AP. Physical illness and treatment of anxiety disorders: A review. In: Zvolensky MJ, Smits J, eds. Series in Anxiety and Related Disorders: Anxiety in Health Behaviors and Physical Illness. New York: Springer, 2008:341-366.

6. Lippa A, Czobor P, Beer B, et al. Selective anxiolysis produced by ocinaplon, a GABAA receptor modulator. Proc Natl Acad Sci 2005;102:7380-7385.

7. Tyrer P. Anxiety: A Multidisciplinary Review. London: Imperial College Press, 1999.

8. Brown TA, Barlow DH. Long-term outcome in cognitive behavioral treatment of panic disorder: Clinical predictors and alternative strategies for assessment. J Consult Clin Psychol 1995;63:754-765.

9. Kite SM, Maher EJ, Anderson K, et al. Development of an aromatherapy service at a cancer centre. Palliat Med 1998;12:171-180.

10. Perry N, Perry E. Aromatherapy in the management of psychiatric disorders. CNS Drugs 2006;20:257-280.

11. Yim WC, Ng KZ, Tsang HWH, Leung AY. A review on the effects of aromatherapy for patients with depressive symptoms. J Altern Complement Med 2009;15:187-195.

12. Glasziou P, Irwig L, Bain CJ, Colditz G. Systematic Reviews in Health Care: A Practical Guide. UK: Cambridge University Press, 2001.

13. Hollis S, Campbell F. What is meant by intention to treat analysis? Survey of published randomised controlled trials. BMJ 1999;319:670-674.

14. Spatz C, Kardas EP. Research Methods in Psychology: Ideas, Techniques, and Reports. New York: McGraw-Hill, 2008.
15. Sjosten N, Kivel S. The effects of physical exercise on depressive symptoms among the aged: A systematic review. Int J Geriatr Psychiatry 2006;21:410-418.

16. Kyle G. Evaluating the effectiveness of aromatherapy in reducing levels of anxiety in palliative care patients: Results of a pilot study. Complement Ther Clin Pract 2006;12: 148-155.

17. Soden K, Vincent K, Craske S, et al. A randomized controlled trial of aromatherapy massage in a hospice setting. Palliat Med 2004;18:87-92.

18. Wilkinson S, Aldridge J, Salmon I, et al. An evaluation of aromatherapy massage in palliative care. Palliat Med 1999;13:409-417.

19. Burnett KM, Solterbeck LA, Strapp CM. Scent and mood state following an anxiety-provoking task. Psychol Rep 2004;95:702-722.

20. Kennedy DO, Little W, Haskell CF. Anxiolytic effects of a combination of Melissa officinalis and Valeriana officinalis during laboratory induced stress. Phytother Res 2006;20: 96-102.

21. Kutlu AK, Yilmaz E, Cecen D. Effects of aroma inhalation on examination anxiety. Teach Learn Nurs 2008;3:125-130.

22. Fujii M, Hatakeyama R, Fukuoka $Y$, et al. Lavender aroma therapy for behavioral and psychological symptoms in dementia patients. Geriatr Gerontol Int 2008;8:136-138.

23. Muzzarelli M, Force M, Sebold M. Aromatherapy and reducing preprocedural anxiety: A controlled prospective study. Gastroenterol Nurs 2006;29:466-471.

24. Lehrner J, Marwinski G, Lehr S, et al. Ambient odors of orange and lavender reduce anxiety and improve mood in a dental office. Physiol Behav 2005;86:92-95.

25. Graham PH, Browne L, Graham J. Inhalation aromatherapy during radiotherapy: Results of a placebo-controlled double-blind randomized trial. J Clin Oncol 2003;21:23722376.

26. Wilkinson SM, Love SB, Westcombe AM, et al. Effectiveness of aromatherapy massage in the management of anxiety and depression in patients with cancer: A multiplecentre randomized controlled trial. J Clin Oncol 2007; 25:532-538.

27. Imura M, Misao H, Ushijima $H$. The psychological effects of aromatherapy-massage in healthy postpartum mothers. J Midwifery Womens Health 2006;51:21-26.

28. Stringer J, Swindell R, Dennis M. Massage in patients undergoing intensive chemotherapy reduces serum cortisol and prolactin. Psycho-Oncology 2008;17:1024-1031.

29. Burns EE, Blamey C, Ersser SJ. An investigation into the use of aromatherapy on intrapartum midwifery practice. J Altern Complement Med 2000;6:141-147.

30. Wiebe EE. A randomized trail of aromatherapy to reduce anxiety before abortion. Eff Clin Pract 2000;3:166-169.

31. Woelk H, Schläfke S. A multi-center, double-blind, randomised study of the lavender oil preparation silexan in comparison to lorazepam for generalized anxiety disorder. Phytomedicine 2010;17:94-99.

32. Hadfield N. The role of aromatherapy massage in reducing anxiety in patients with malignant brain tumors. Int J Palliat Nurs 2001;7:279-285.

33. Fountoulakis KN. Reliability and psychometric properties of the Greek translation of the state-trait anxiety inventory form Y: Preliminary data. Ann Gen Psychiatry 2006; 5:2.

34. Vizi ES. Handbook of Neurochemistry and Molecular Neurobiology. NewYork: Springer, 2008. 
35. Lis-Balchin M, Hart S. Studies on the mode of action of the essential oil of lavender (Lavandula angustifolia P. Miller) Phytother Res 1999;13:540-542.

36. Umezu T. Behavioral effects of plant-derived essential oils in the geller type conflict test in mice. Jpn J Pharmacol 2000;83:150.

37. Cooke B, Ernst E. Aromatherapy: A systematic review. Br J Gen Pract 2000;50:493-496.
Address correspondence to: Hector W.H. Tsang, PhD

Neuropsychiatric Rehabilitation Laboratory Department of Rehabilitation Sciences The Hong Kong Polytechnic University Hung Hom, Hong Kong

E-mail: rshtsang@inet.polyu.edu.hk 